\title{
Conferência
}

\section{Procedimentos cirúrgicos ou hemodinâmicos? A visão do cirurgião*}

\author{
Domingo M. BRAILE
}

RBCCV 44205-490

Vamos falar hoje sobre a visão do cirurgião diante dos procedimentos hemodinâmicos ou cirúrgicos. Farei uma pequena introdução, que é metodológica, e acho muito importante para que possamos discutir com nossos colegas.

Os estudos randomizados que têm sido feitos entre a dilatação e a cirurgia de coronária são em grande número. Todos nós já ouvimos falar do EAST, ERACI, RITA, GABI, CABRI e BARI, mas existem muitos outros. Há um livro com cerca de 400 páginas só com a citação dos estudos comparativos. $\mathrm{Na}$ realidade, nenhum procedimento foi mais estudado, por métodos estatísticos, principalmente, randomizados, do que a revascularização do miocárdio.

Existe, na metodologia científica, um conceito que se chama continuidade. Este é o conceito chave na investigação clínica e nós estamos muito acostumados com ele. Permite fazer inferências sobre pacientes, em grupos ou individualmente, mas não informa em quem ou quando o evento ocorrerá. Quando temos uma curva atuarial, que é o exemplo mais clássico deste tipo de continuidade, podemos dizer que teremos $80 \%$ dos pacientes vivos em 5 anos, mas não sabemos qual paciente constará desta estatística. Assim, as curvas atuariais são muito importantes como exemplo de continuidade, que se contrapõe à Medicina Baseada em Evidência. É a evidência versus a prova, que é diferente da fé. Podemos ter fé em um procedimento, mas evidência só mediante prova, e prova só pode ser obtida com Medicina Baseada em Evidência.
Um outro conceito, que foi apresentado por $\mathrm{EH}$ Blackstone, em 1999, é que descrição é diferente de previsão: "uma coisa é descrever o fenômeno que se observa, é uma análise descritiva dos dados". Provavelmente, durante este nosso congresso tenhamos ouvido muito sobre descrição de um fenômeno e como ele se desenvolve, porém é completamente diferente de "analisar, sintetizar e apresentar o conhecimento obtido para que seja útil em prever o resultado esperado em um novo grupo de pacientes"; isto é Medicina Baseada em Evidência. A Medicina Baseada em Evidência está mudando, inclusive, a maneira de se ensinar Medicina, que é através do PBL - problem based learning. Acho que estes conceitos são importantes para nós e temos que tentar absorvê-los da melhor forma, para que possamos nos defender.

As previsões e comparações são assunto complexo, principalmente na revascularização miocárdica, porque existem muitos fatores de risco independentes, já que recomendações para um paciente específico exigem base na sua combinação particular de fatores de risco e no risco inerente ao procedimento. Então, nós estamos convictos de que, se não realizarmos cirurgia de coronária com baixa mortalidade, não adianta operar, já que a doença é menos grave que a mortalidade que, eventualmente, possa ocorrer. Todos sabem que a cirurgia da estenose aórtica assintomática é a principal causa de morte imediata nessa doença, porque o indivíduo assintomático vive muitos anos e, se nós operarmos e por azar o paciente morrer, é um desastre.

Conferência proferida no $27^{\circ}$ Congresso Nacional de Cirurgia Cardíaca. Rio de Janeiro, RJ, 23 a 25 de março, 2000.

* Transcrição de fita gravada.

Prof. Dr. Livre Docente, Titular de Cirurgia Cardíaca da Faculdade Estadual de Medicina de São José do Rio Preto. Prof. de Cirurgia Cardíaca da Faculdade de Medicina da Universidade Estadual de Campinas. Membro Titular da Sociedade Brasileira de Cirurgia Cardiovascular. Membro efetivo da American Association of Thoracic Surgery.

Endereço para correspondência: Domingo M. Braile. Av. Juscelino Kubitschek, 3.101. São José do Rio Preto, SP, Brasil. CEP 15091-450. Tel: (017) 210-7000.e-mail: domingo@braile.com.br 
O número teórico mínimo de possibilidades combinatórias para análise de um único evento coronariano seria:

- Número de vasos comprometidos (de 1 a 4 )

- Função ventricular esquerda comprometida (abaixo de 20, de 20-30, de 30-40 ou superior a 40)

- Gravidade da isquemia (segundo a Canadian Association, em I, II, III e IV)

- Presença ou não de infarto prévio

- Presença ou não de hipertensão

- Presença ou não de diabete

- Formas de tratamento (clínico, cirúrgico ou através de cateterismo intervencionista)

Se fizermos a conta, teremos $1.536(4 \times 4 \times 4$ $\times 2 \times 2 \times 2 \times 3=1.536)$ possibilidades combinatórias para análise de um único evento; então, é muito difícil fazer a avaliação de um evento usando a análise combinatória.

Vou dar um exemplo de variáveis independentes, também chamadas de variáveis explanatórias, preditoras ou co-variáveis. No estudo EAST, a idade média dos pacientes era de 61 anos. Assim sendo, este estudo não é aplicável a pacientes com 30 anos. Também não é um bom estudo para sabermos o que acontece com as mulheres, já que $73 \%$ dos pacientes era do sexo masculino. Com relação ao número de vasos comprometidos, $60 \%$ dos pacientes apresentavam lesão em 2 vasos e $40 \%$ em 3; nenhum doente apresentava lesão em 1 ou 4 vasos. A fração de ejeção média no estudo EAST era 0,60 e raramente operamos um doente com este índice. Apenas $41 \%$ dos doentes apresenta-vam infarto prévio. Geralmente operamos muito mais pacientes nesta condição. Noventa e um por cento dos pacientes tinham angina classe II e III, restando muito poucos em classe IV. Finalmente, apenas $23 \%$ dos pacientes tinham diabete. Este exemplo serve para mostrar que não é possível encaixar este estudo EAST em um doente que chega ao consultório, sem que sejam avaliadas exatamente todas estas variáveis.

John Graunt morava em Londres no período do ressurgimento da Peste Negra, em 1603, e, apesar de ser apenas um almoxarife, era um indivíduo muito inteligente que criou o conceito de Hazard Function. Hazard Function era uma expressão usada, na época, em jogos de dados; hazard que dizer azar, sorte, acaso, perigo, risco. Esse indivíduo estabeleceu uma relação entre o estoque que havia na prateleira e a entrada e saída de mercadorias. Baseado nessas informações, ele poderia saber qual era o seu estoque. Deduziu, daí, que não adiantaria as pessoas ficarem apenas contando os mortos, o que era feito pelos padres da época; era preciso saber por que as pessoas morriam. Foi quando surgiu o $\lambda$. Se eu tenho um grupo em risco e se eu souber o risco dessas pessoas morrerem $(\lambda)$, então tenho como saber o provável número de mortos. E ele percebeu que as pessoas que tinham animais em casa contraíam mais peste. $E$, também, quando chegava um navio a Londres, aumentava o número de mortos pela peste. Com isto, ele criou o Hazard Rate: que permite, conhecendo a possibilidade de risco, prever a mortalidade (Figura 1). Deste trabalho resultou o Hazard Ratio, o qual originou o Risco Relativo, o ODDS Ratio e o log ODDS e, finalmente, a Regressão Logística.

Kirklin \& Barrat-Boyes (1993), em seu livro clássico, afirmam que o único modo confiável de se fazer predições e comparações paciente-específicas é por meio das equações com análise multivariada dos fatores de risco. Fica claro que temos que trabalhar com análise multivariada de fatores de risco, senão estaremos sempre nos baseando na fé e na imaginação. Predições e comparações paciente-específicas são difíceis porque exigem altos recursos de computação, que, por sorte, estão chegando às nossas mãos.

O que nós temos que saber afinal? É simples; temos que saber a probabilidade da ocorrência de um evento, antes da intervenção. E como saber? Com o auxílio de uma simples fórmula de probabilidade, podemos construir a curva Logit Units, a qual nos dá a probabilidade de um evento (Figura 2).

Com isto podemos, por exemplo - se chegar um indivíduo com insuficiência cardíaca, hoje, ao hospital, após medido o índice cardíaco desse paciente e com a fórmula da probabilidade da regres-

MODELO DO PROCESSO NASCIMENTO-MORTE

(ANÁLISE DE EVENTOS TEMPO-RELACIONADOS)

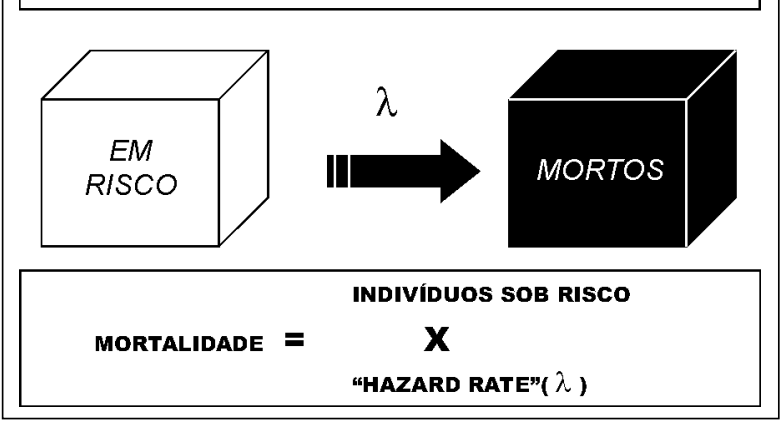

Fig. 1 - Esquema da análise de eventos tempo-relacionados. (Fonte: Kirklin J W \& Barrat-Boyes B G. Cardiac surgery. 2.ed. New York: Churchill Livingstone, 1993. v.1). 


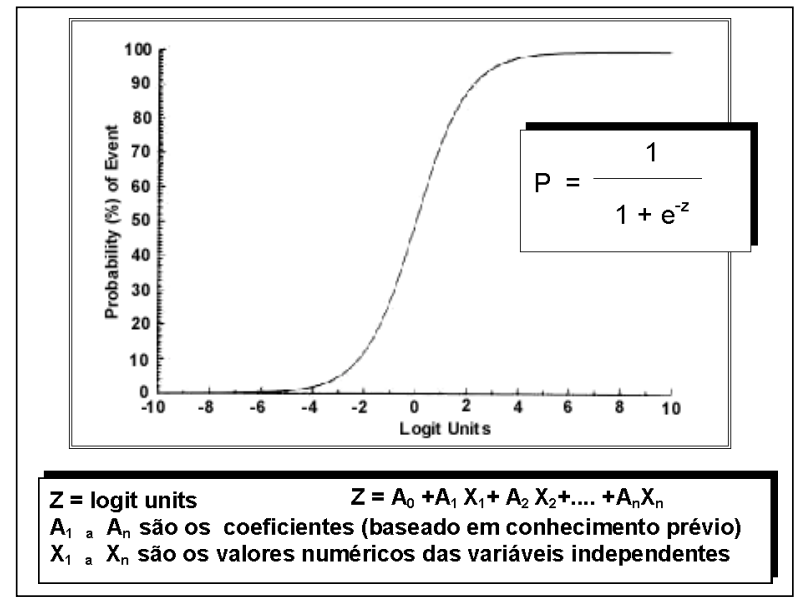

Fig. 2 - Curva Logit Units.

(Fonte: Kirklin J W \& Barrat-Boyes B G. Cardiac surgery. 2.ed. New York: Churchill Livingstone, 1993. v.1).

são logística - dizer que, se ele tiver 2 litros por minuto por $\mathrm{m}^{2}$ de débito cardíaco, a chance do paciente morrer é de $10 \%$ e, se ele tiver 1 litro de índice cardíaco, a chance eleva-se para $85-90 \%$. Não é tão difícil de entender e temos que dominar este tipo de raciocínio para que possamos discutir com os outros colegas (Figura 3).

Os próximos exemplos são baseados em um banco de dados da KUL - Katholleke Universiteit in Leuven, na Bélgica, de uma casuística de 10.000 pacientes que foram estudados durante 10 anos com tratamento clínico, com angioplastia e com cirurgia.

\section{Caso 1}

Chega ao nosso consultório um homem com 63 anos, com angina estável discreta, pressão arterial

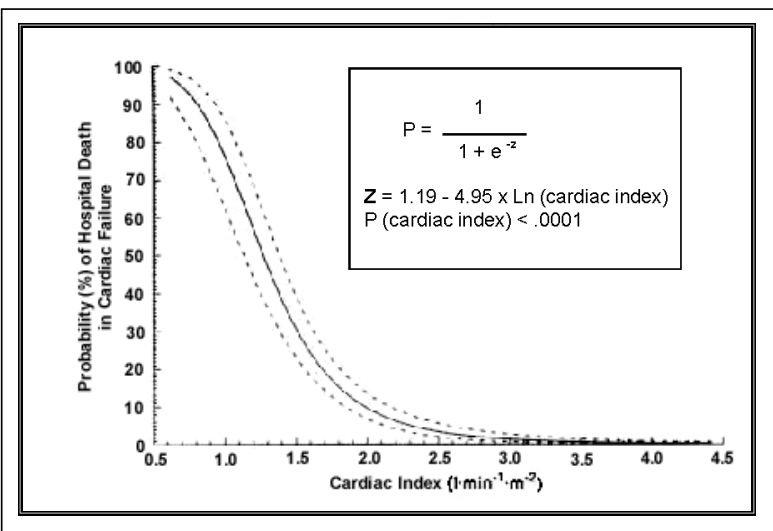

Fig. 3 - Probabilidade de óbito hospitalar na insuficiência cardíaca $x$ índice cardíaco.

(Fonte: Kirklin J W \& Barrat-Boyes B G. Cardiac surgery. 2.ed. New York: Churchill Livingstone, 1993. v.1). normal, normolipêmico, teste ergométrico levemente positivo e com obstrução de $70 \%$ em terço proximal da artéria interventricular anterior (DA), com fração de ejeção de 0,60. O que fazer com esse indivíduo? A chance desse paciente sobreviver ao longo de 10 anos, baseada na estatística de 10.000 pacientes, é praticamente a mesma se ele for tratado com medicação (80\%), com angioplastia (85\%) ou se for submetido a cirurgia (91\%) - Figura 4A.

Este homem retornou com 64 anos, com angina classe IV, o teste ergométrico ficou muito positivo, com lesão $80 \%$ na artéria interventricular anterior e com fração de ejeção boa $(0,60)$. Qual é a chance desse indivíduo sobreviver? Comparando-se as vantagens da cirurgia às da angioplastia, verificamos que existem poucas vantagens do procedimento cirúrgico. Então, a esse indivíduo nós temos que indicar angioplastia, porque não existe consistência estatística para indicar outro tipo de tratamento (Figura 4B).

Nosso paciente continuou vivendo, volta agora com 67,5 anos. Ele fez uma angioplastia há 3 anos, teve um infarto há 6 meses, angina classe IV, obstrução de artéria interventricular anterior de $80 \%$ e de coronária direita de $70 \%$ e com uma fração de ejeção que, devido ao infarto, baixou para 0,30. Qual é a chance de sobrevivência em 5 e 10 anos? Praticamente não existe muita diferença entre os procedimentos, seja clínico, angioplastia ou cirúrgico (Figura 4C). Comparando-se um procedimento com outro, se nós operarmos o paciente ou fizermos angioplastia, o benefício será similar. Indicou-se a esse paciente outra angioplastia.

Aos 69,5 anos, esse homem está com 2 angioplastias prévias (5 e 2 anos), infarto há 5,5 anos, angina classe IV, com obstruções na artéria interventricular anterior (80\%), coronária direita $(70 \%)$ e marginal $(70 \%)$ e a fração de ejeção se mantém em 0,30 . Se submetermos esse paciente a uma nova angioplastia, ele terá $27 \%$ de chance de sobreviver em 10 anos e, se fizermos um tratamento clínico, ele terá $45 \%$. No entanto, se for operado, terá $62 \%$ de chance de viver 10 anos e $88 \%$ de viver 5 anos. Comparando-se um tratamento com outro, observamos que a cirurgia é melhor que o tratamento clínico e a angioplastia. Este tipo de estudo nos dá argumentos para discutir com os clínicos. Através dele podemos provar que há pacientes que são candidatos à cirurgia, como esse indivíduo, que só pode ser beneficiado pelo procedimento cirúrgico (Figura 4D).

\section{Caso 2}

Mulher de 70 anos, portadora de angina instável, apresentando lesão de artéria interventricular anterior de $85 \%$ em terço médio, de coronária direita $(70 \%)$ e de marginal $(60 \%)$, com fração de ejeção 

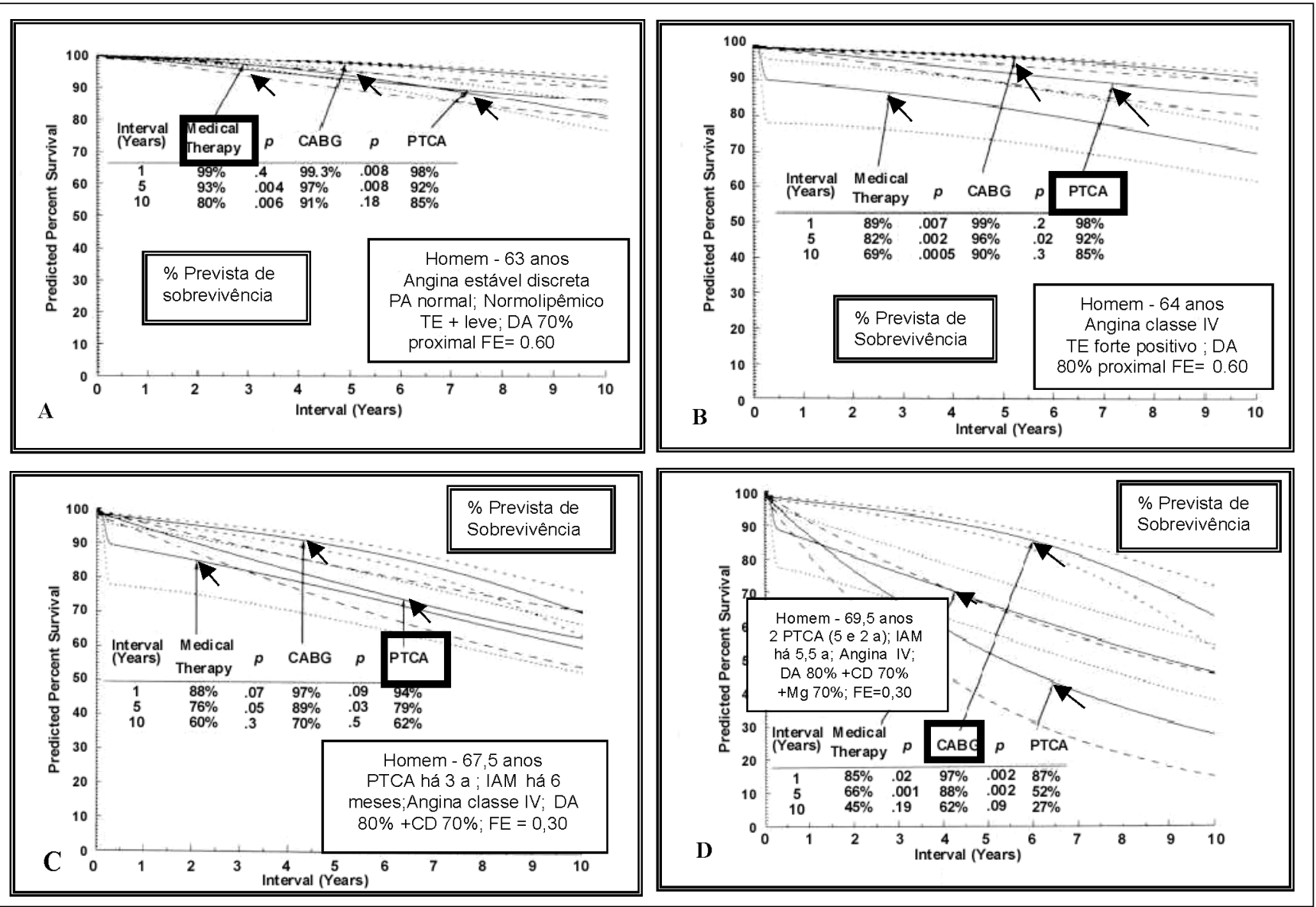

Fig. 4 - Curvas relacionadas ao caso 1.

(Fonte: Kirklin J W \& Barrat-Boyes B G. Cardiac surgery. 2.ed. New York: Churchill Livingstone, 1993. v.1).

de 0,60. Qual é a chance de a paciente ficar livre de revascularização? Se for tratada clinicamente, ela tem uma chance de $38 \%$ de ficar livre de revascularização; se for submetida a angioplastia, a chance cai para $13 \%$, em 10 anos (Figura $5 \mathrm{~A}$ ). Comparando-se os métodos quanto aos seus benefícios, podemos verificar que, para essa paciente, os tratamentos clínico ou cirúrgico são praticamente iguais e que a angioplastia não trará benefícios; mantémse, então, o tratamento clínico (Figura 5B). Quanto à sobrevivência prevista, o tratamento cirúrgico apresenta resultados ligeiramente superiores ao clínico, mas significativamente melhores em relação à angioplastia (Figura $5 \mathrm{C}$ ). Se comparamos um método com outro, observamos que a operação e a terapêutica clínica apresentam resultados muito semelhantes; no entanto, para essa paciente a cirurgia é muito melhor que a angioplastia (Figura 5D).

\section{Caso 3}

Homem de 66 anos portador de angina leve estável com infarto há 2 anos, classe funcional II.
Teste ergométrico positivo, obstrução de $80 \%$ da DA, $30 \%$ da coronária direita e $30 \%$ da $\mathrm{Mg}$, tendo uma fração de ejeção muito baixa de $20 \%$. Podemos observar que, neste caso, o tratamento clínico ou a cirurgia dariam o mesmo resultado para a sobrevivência em 5 e 10 anos (Figura 6A). Contudo, se fosse submetido a angioplastia o resultado seria muito pior, com a sobrevivência de cerca de $30 \%$ em 1,5 ou 10 anos. Nesse mesmo senhor aos 67 anos, agora com infarto há 3 anos e angina classe IV, com as mesmas obstruções, a diferença entre o tratamento cirúrgico e o tratamento clínico se distancia muito. Se submetermos esse paciente ao procedimento cirúrgico, ele tem $66 \%$ de chance de sobrevivência em 10 anos e $86 \%$ de chance de viver 5 anos. Com o tratamento clínico, esta chance cai para $43 \%$ e $65 \%$, respectivamente. Então, nesse indivíduo está indicada a cirurgia (Figura 6B).

\section{Principais Constatações: Angioplastia x Cirurgia}

As principais constatações em relação à angioplastia versus a cirurgia, em doença multiarterial, nos diferentes estudos, são as seguintes: 


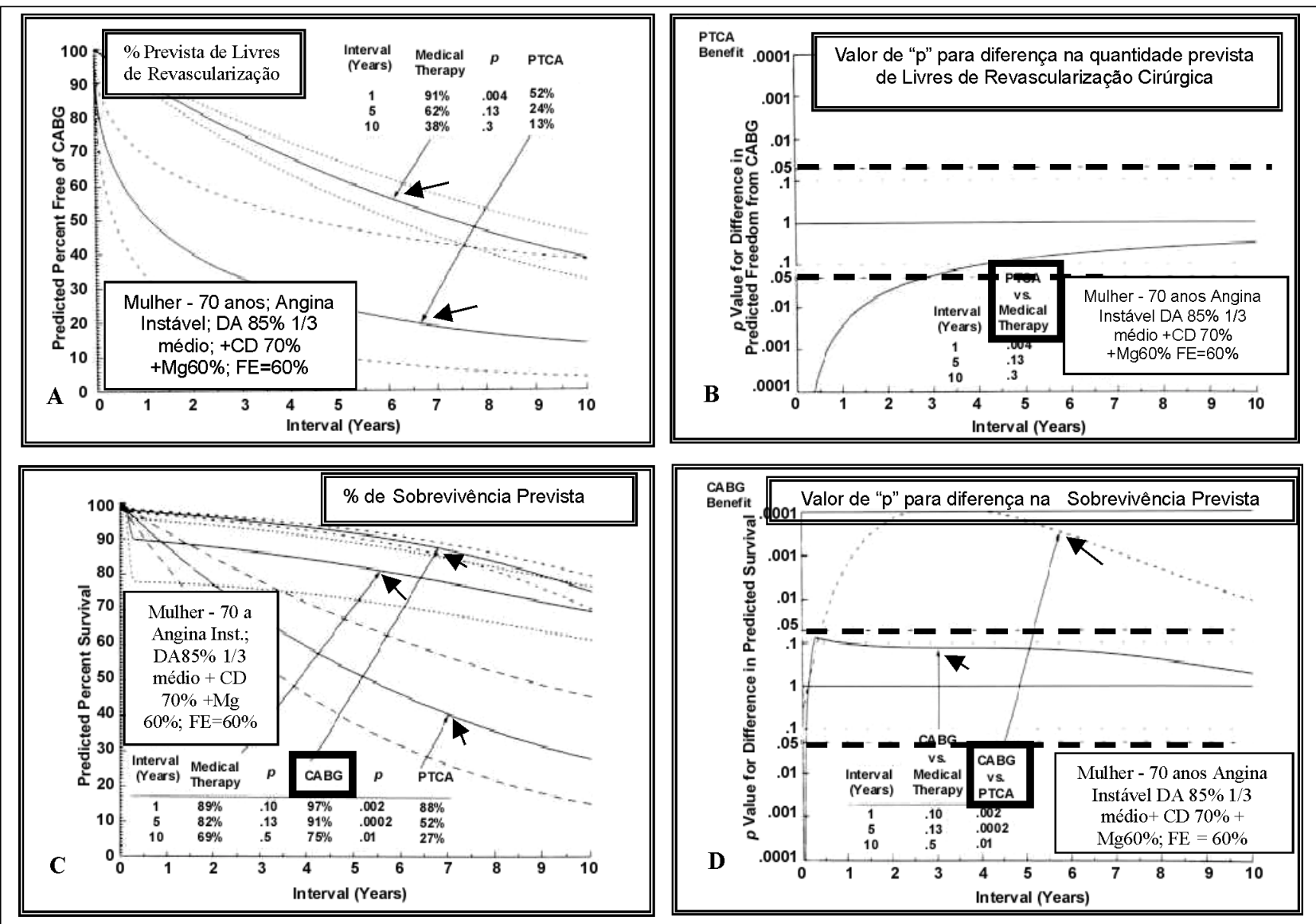

Fig. 5 - Curvas relacionadas ao caso 2 .

(Fonte: Kirklin J W \& Barrat-Boyes B G. Cardiac surgery. 2.ed. New York: Churchill Livingstone, 1993. v.1).
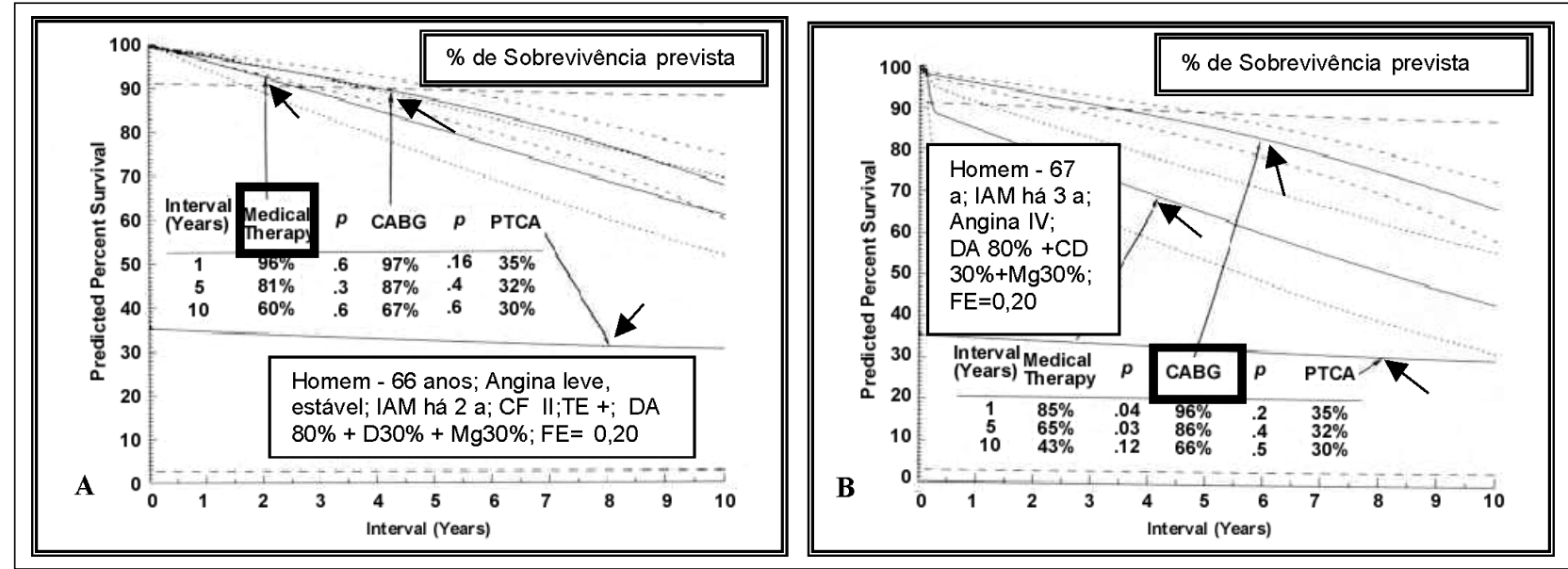

Fig. 6 - Curvas relacionadas ao caso 3.

(Fonte: Kirklin J W \& Barrat-Boyes B G. Cardiac surgery. 2.ed. New York: Churchill Livingstone, 1993. v.1).

BARI (Cirurgia) * Em pacientes diabéticos há menor mortalidade e menos reintervenção quando se usa a artéria torácica interna.
* Nas mulheres, há maior sobrevivência após 5 anos.

EAST

* Não houve diferença na sobrevivência após 5 anos. 
* Mais reintervenção após angioplastia. Sem diferença nos custos após 3 anos.

RITA

* Sobrevivência similar após 6,5 anos.Mais reintervenção e retorno da angina após angioplastia.

* Sem diferença de custo após 5 anos.

Estes estudos, randomizados, são também extremamente limitados; o RITA, O EAST e o BARI selecionaram 54.093 e, destes, só puderam randomizar 3.232 (Tabela 1). Em todos estes estudos a média de randomização é de apenas $4 \%$ a $8 \%$ dos pacientes para estudo comparativo, porque os demais não se encaixam nas premissas, nos protocolos, ou seja, estão fora da realidade. É preciso ter muito cuidado ao ler um estudo randomizado, pois um grande número de doentes é selecionado, mas apenas uma pequena parcela é randomizada. Será que o nosso doente encaixa-se nos 54.093 ou nos 3.232 ?

TABELA 1

\begin{tabular}{|c|c|c|}
\hline \multicolumn{3}{|c|}{ LIMITAÇÃO DOS ESTUDOS RANDOMIZADOS } \\
\hline ESTUDO & SELECIONADOS & RANDOMIZADOS \\
\hline RITA & 27975 & 1011 \\
\hline EAST & 5118 & 392 \\
\hline BARI & 21000 & 1829 \\
\hline TOTAL & 54093 & 3232 \\
\hline
\end{tabular}

Tivemos um paciente, sexo masculino com 42 anos, portador de angina estável, pressão arterial normal, dislipidêmico, teste de esforço positivo, com lesão de $80 \%$ em terço proximal da artéria interventricular anterior e de $60 \%$ na circunflexa, com fração de ejeção de 0,85 . O hemodinamicista sugeriu que fosse realizada uma angioplastia e colocou um stent na artéria interventricular anterior. Três meses após a angioplastia e colocação de stent, o pacien- te apresentou dor precordial intensa após esforço e infarto do miocárdio. Ao cateterismo, a artéria interventicular anterior ficou completamente ocluída e com reenchimento pobre pela coronária direita; o ventrículo desse paciente, que era absolutamente normal, ficou com um aneurisma.

É importante ressaltar que não basta só a meIhor evidência; é necessário considerar o paciente individualmente, ou seja, considerar a melhor evidência existente na literatura adaptada à condição desse paciente.

À guisa de conclusão, podemos dizer que a angioplastia versus a cirurgia, em lesões multiarteriais, tem sobrevivência e custos similares. Temos que considerar, de forma individualizada, a natureza mais invasiva da cirurgia e o maior risco de angina recorrente e de reintervenções com a angioplastia. Além disto, não podemos desconsiderar o tratamento clínico, porque, hoje, há a possibilidade de uso de potentes medicamentos, o betabloqueador foi uma grande conquista que não podemos de maneira nenhuma esquecer. Os stents e os enxertos arteriais múltiplos dependem de estudos prospectivos a longo prazo. As estatisticas apresentadas para stent multiarterial referem-se a um período médio de 6 meses a 1 ano, no máximo. Da mesma forma, os nossos enxertos arteriais ainda precisam ser estudados, não há evidências de que sejam muito melhores que a veia safena. Nós só sabemos, por enquanto, que a artéria torácica interna para a interventricular anterior é muito boa, porém, para além disto, ainda temos que continuar estudando, o que é um vasto campo de pesquisa.

Hipócrates dizia "Sedare dolorem opus divinum est". Nossa função como médicos é sedar dores; se o indivíduo tem sintomas, estamos autorizados a usar a nossa experiência e individualizar aquela pessoa, estabelecendo qual o melhor tratamento para ela. O que a Natureza faz? Ela faz colaterais. E nós cirurgiões somos capazes de imitar a Natureza. Na realidade, a Natureza faz pontes, não faz stents...

DESCRITORES: Ensaio clínico randomizado. Epidemiologia. Medicina baseada em evidências. Fatores de risco. Probabilidade. Modelos logísticos. Revascularização miocárdica. Cardiopatias, cirurgia. Análise de sobrevivência. Angioplastia. Hemodinâmica. Cardiopatias, resultado de tratamento.

DESCRIPTORS: Randomized controlled clinical trial. Epidemiology. Evidence-based Medicine. Risk factors. Probability. Logistic, guides. Myocardial revascularization. Heart diseases, surgery. Survival analysis. Angioplasty. Hemodynamics. Heart diseases, treatment outcome. 